\section{Reinke's Edema: investigations on the role of MIB-1 and hepatocyte growth factor}

\author{
M. Artico, ${ }^{1}$ E. Bronzetti, ${ }^{2}$ B. Ionta, \\ M. Bruno, ${ }^{1}$ A. Greco, ${ }^{1}$ G. Ruoppolo, ${ }^{1}$ \\ A. De Virgilio, ${ }^{1}$ L. Longo, \\ M. De Vincentiis' \\ 'Department of Otorhinolaringology, \\ Audiology and Phoniatry G. Ferreri; \\ 2Department of Human Anatomy, \\ University of Rome "La Sapienza", Rome, \\ Italy
}

\section{Abstract}

Reinke's edema is a benign disease of the human vocal fold, which mainly affects the sub-epithelial layer of the vocal fold. Microscopic observations show a strongly oedematous epithelium with loosened intercellular junctions, a disruption of the extracellular connections between mucosal epithelium and connective tissue, closely adherent to the thyroarytenoid muscle. Thickening of the basal layer of epithelium, known as Reinke's space, high deposition of fibronectin and chronic inflammatory infiltration it is also visible. We analyzed, together with the hepatocyte growth factor (HGF), the expression level of MIB-1 in samples harvested from patients affected by Reinke's edema, in order to define its biological role and consider it as a possible prognostic factor in the follow-up after surgical treatment. We observed a moderate expression of HGF in the lamina propria of the human vocal fold and in the basal membrane of the mucosal epithelium. Our finding suggests that this growth factor acts as an anti - fibrotic agent in Reinke's space and affects the fibronectin deposition in the lamina propria. MIB-1, on the contrary, showed a weak expression in the basement membrane of the mucosal epithelium and a total absence in the lamina propria deep layer, thus suggesting that only the superficial layer is actively involved in the reparatory process with a high regenerative capacity, together with a high deposition of fibronectin. The latter is necessary for the cellular connections reconstruction, after the inflammatory infiltration.

\section{Introduction}

The human vocal fold can be divided into five different layers: squamous epithelium, lamina propria (superficial, intermediate and deep layers) and the thyroarytenoid muscle..$^{1-3}$

The lamina propria is uniquely responsible for vibration of the vocal fold. ${ }^{4-6}$ The superficial layer of the lamina propria, also known as Reinke's Space, plays a relevant role in production and quality of the voice, since its tension and structural integrity determine the vibratory characteristics of the human vocal fold. Madruga de Melo et al. ${ }^{4}$ described the specific orientation of collagen type I and III in the lamina propria. These authors report a narrow band of type I collagen in the superficial layer, a middle layer composed of type III and, within the muscle into the deep, an intertwining of types I and III. Collagen assessment in the normal vocal folds was defined by Madruga de Melo et al. ${ }^{4}$ as an intertwined network forming a wicker basket, necessary to stabilize the lamina propria of the human vocal fold during vibration. Collagen type I and III (also known as reticular fibres), ${ }^{5}$ mainly constitute the fibrillar collagen network in the human vocal fold lamina propria. ${ }^{4,5}$ The arrangement of collagen fibres in the lamina propria of the human vocal fold confers resistance to muscle contraction and allows elongation in voice modulation, particularly in the superficial layer, the most important compartment involved during vibratory function. Any alteration in the composition of the extracellular matrix (ECM) of Reinke's space causes changes in quality of vocal fold function, leading to pathological phenomena such as disphonia or dispnea.

Reinke's edema is a benign lesion characterized by swelling of the non-muscle compartment of the vocal fold situated beneath its surface lining, especially in the superficial layer of the lamina propria. This swelling leads to Reinke's space being enriched with an excess of exudative fluid drained from the sub-epithelial tissue to the epithelium: this determines widening of the intercellular compartments and spreading out of inflammatory cells and fibroblasts, thus reducing vocal fold vibration and quality of voice production. ${ }^{2,8-12}$ Microscopic investigations show an oedematous epithelium with loosened intercellular junctions and widening of intercellular spaces, especially in the basal membrane. ${ }^{13}$ The most frequent aetiological factor in Reinke's edema is smoking. ${ }^{14}$ In fact, all the patients we examined were found to be heavy smokers.

As we previously described ${ }^{15} \mathrm{Ki} 67$ is a cell proliferation marker, utilized as a prognostic factor in the follow up of many neoplastic lesions, therefore our purpose was to analyze the expression level of MIB-1 (anti-Ki67) in the Reinke's edema samples, with the purpose of evaluate its biological role in this disease and its diagnostic application.

The hepatocyte growth factor (HGF) is a cellular growth, motility and morphogenetic factor acting primarily upon epithelial cells and
Correspondence: Marco Artico, M.D., Pharm. D., Dept. of Otorhinolaringology, Audiology and Phoniatry "G. Ferreri", University of Rome "La Sapienza", p.le Aldo Moro 5, 00185 Rome, Italy. Tel: +39.06.4997675 Fax: +39.06.49976817.

E-mail:marco.artico@uniromal.it

Key words: Reinke's edema, Ki67, HGF, fibronectin, immunohistochemistry, extracellular matrix.

Received for publication: 27 January 2010. Accepted for publication: 30 April 2010.

This work is licensed under a Creative Commons Attribution 3.0 License (by-nc 3.0).

(C) Copyright M. Artico et al., 2010

Licensee PAGEPress, Italy

European Journal of Histochemistry 2010; 54:e30 doi:10.4081/ejh.2010.e30

endothelial cells, but also on haemopoietic progenitor cells. ${ }^{16}$ It has a major role in embryonic organ development, in adult organ regeneration and in wound healing, with a strong antifibrotic action, improving hyaluronic acid production and decreasing collagen release by fibroblasts in Reinke's space. This occurrence leads to morphological and functional changes in this cell population. ${ }^{1718}$

Hence, the final objective of our experimental study was to analyze the functional role and the localization of MIB-1 (anti-Ki67) in the oedematous tissue, in order to consider it as a possible prognostic marker after surgical treatment and to ascertain a possible correlation between MIB-1 (anti-Ki67) and HGF in the development of Reinke's Edema.

\section{Materials and Methods}

\section{Patients}

We analyzed the surgical biopsies of 15 patients with Reinke's edema and 5 samples from normal vocal (referred to as negative controls) and 5 samples as positive controls (brain, melanoma, liver). Reinke's edema samples and negative control tissues were surgically removed from the patients and processed for immunohistochemical analysis. Experiments were performed in compliance with the Italian laws and guidelines concerning the informed consent of the patients. The following molecules were investigated: HGF, fibronectin and MIB-1 (Ki67 antigen).

\section{Immunohistochemical analysis}

Surgical samples of Reinke's edema were washed in PBS, fixed in 10\% formalin and rou- 
tinely embedded in paraffin. Serial $5 \mu \mathrm{m}$ thick sections were obtained using a rotatory microtome, mounted on gelatin-coated slides and processed for immunohistochemistry. The following antibodies were used: i) HGF, a rabbit polyclonal antibody raised against amino acids 32-176 of HGF $\alpha$ of human origin. (Santa Cruz, CA, USA, at a final dilution 1:100); ii) fibronectin, a mouse monoclonal antibody raised against a region in the ED-A domain of cellular Fibronectin of human origin (Santa Cruz, CA, USA, at a final dilution 1:100); iii) MIB-1, a rabbit polyclonal antibody raised against amino acids 2641-2940 mapping at the C-terminus of Ki-67 of human origin (Santa Cruz, CA, USA, at a final dilution 1:100). Incubation with primary antibodies was performed overnight at $4^{\circ} \mathrm{C}$ at a final concentration of $2-5 \mu \mathrm{g} / \mathrm{mL}$. Optimal antisera dilutions and incubation times were assessed in a series of preliminary experiments. After exposure to the primary antibodies, slides were rinsed twice in phosphate buffer and then incubated ( $1 \mathrm{~h}$ and $30 \mathrm{~min}$ at room temperature) with the appropriate secondary antibodies against rabbit (anti-rabbit for Fibronectin and HGF immunohistochemistry; Boehringer Mannheim GmbH, Mannheim, Germany) and against mouse (anti-mouse for MIB-1/Ki67 immunohistochemistry; Sigma Chemical Co., St. Louis, M0, USA), conjugated to horseradish peroxidase (HRP) at a final dilution 1:100). After a further wash with phosphate buffer, slides were treated with $0.05 \%$ 3,3-diaminobenzidine and $0.1 \% \mathrm{H}_{2} \mathrm{O}_{2}$. Finally, sections were counterstained with Mayer's hematoxylin and observed by using a light microscope. In order to block endogenous peroxidase activity, slides were pre-treated with $3 \% \mathrm{H}_{2} \mathrm{O}_{2}$, whereas the non-specific binding of immunoglobulin to glass and tissue (background) was prevented by adding $3 \%$ fetal calf serum to the incubation medium. Negative control experiments were done: i) by omitting the primary antibody; ii) by substituting the primary antibody with equivalent amount of non-specific immunoglobulin; iii) by pre-incubating the primary antibody with the specific blocking peptide (antigen/antibody $=5$ according to customer's instructions).

\section{Results}

\section{Immunohistochemistry}

Immunoreactivity for fibronectin, MIB-1 and HGF was described in Reinke's edema samples, compared to the negative controls (normal vocal fold biopsies) and positive controls (data not shown) (Table 1). In Reinke's edema samples, HGF showed a moderate expression

Table 1. Reinke's edema.

\begin{tabular}{lccc} 
& Fibronectin & HGF & MIB-1 \\
Epithelium & + & \pm & \pm \\
Lamina propria & +++ & + & \\
\hline Endothelium & ++ & + & \\
\hline
\end{tabular}

\pm : weak immunoreactivity; +: moderate immunoreactivity; ++: relevant immunoreactivity; +++: strong immunoreactivity.
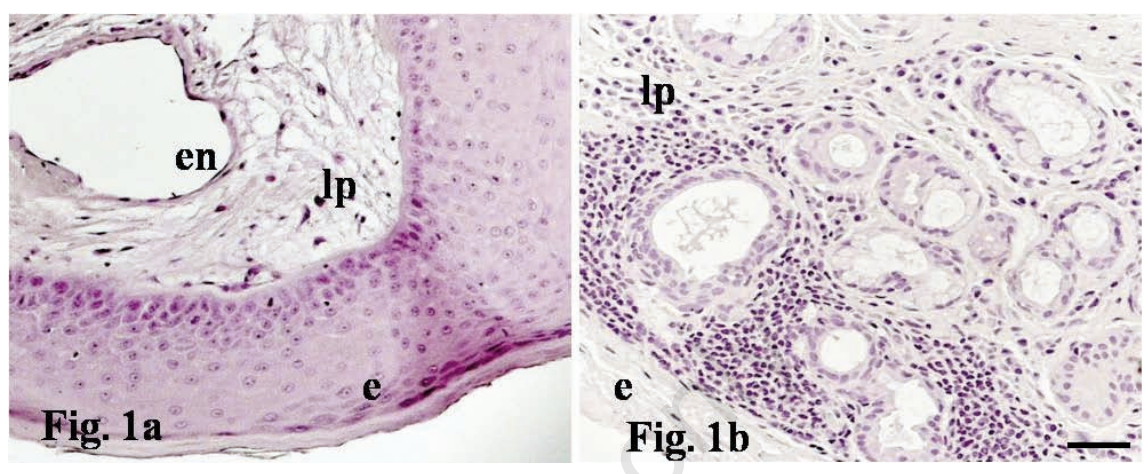

Figure 1. a. Micrograph of representative HGF immunostaining in sections of Reinke's edema. HGF shows a weak expression level in the mucosal epithelium (e); it is also visible a moderate expression signal in the lamina propria (lp), as revealed on the endothelial layer (en). b. Micrograph of human normal vocal fold, considered as negative control. Bar: $25 \mu \mathrm{m}$.
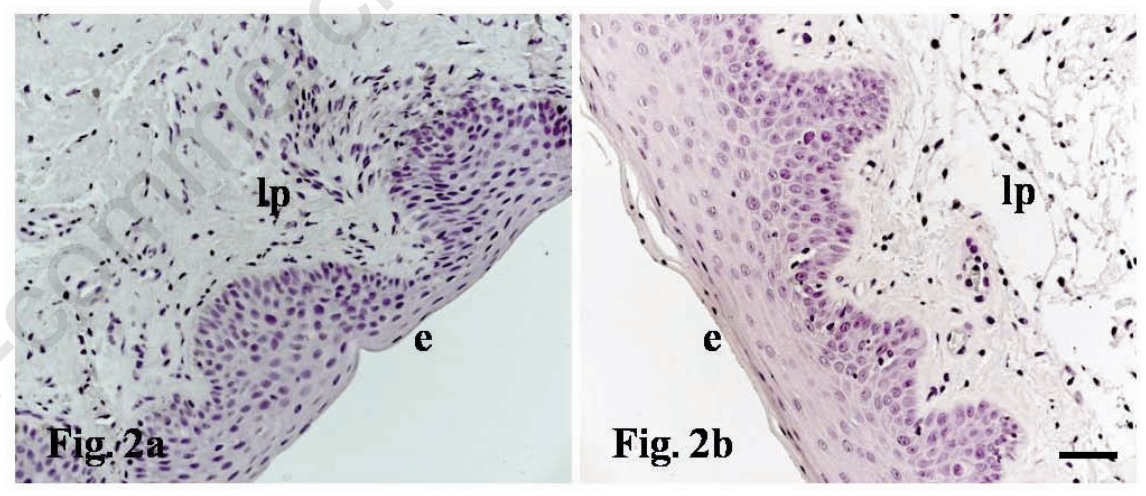

Figure 2. a. Micrograph of representative MIB-1 immunostaining in sections of Reinke's edema. MIB-1 (anti Ki67) demonstrates a weak expression in the mucosal epithelium (e), while a total absence in the lamina propria (lp) and on the vessel endothelium (en). $b$. Micrograph of human normal vocal fold, considered as negative control. Bar: $25 \mu \mathrm{m}$.

(+) in the lamina propria (lp) superficial and intermediate layers, as revealed in the endothelial cells $(+)(\mathrm{en})$ of the human vocal fold (Figure 1a), while a weak level (+/-) was observed in the epithelium (e), thus confirming its anti - fibrotic activity (Figure 1a).

Figure 1b shows the human normal vocal fold, considered as the negative control. MIB-1 (anti-Ki67) in Reinke's edema samples revealed a weak expression (+/-) in the mucosal epithelial layer (e), but a total absence in the lamina propria (lp) superficial and intermediate layers, as well as in vessels endothelium (en): this suggests that only the basal membrane is active in the reparative process with high regenerative capacity, together with a high fibronectin deposition (Figure 2a). In Figure 2b we showed the human normal vocal fold, considered as the negative control. At last, Reinke's edema samples revealed a strong fibronectin expression $(+++)$ in the lamina propria (lp) superficial and intermediate layers, relevant $(++)$ in the endothelium (en), while a moderate immunostaining (+) was observed in the epithelium (e) (Figure $3 \mathrm{a}$ ), as in the normal vocal fold, 
thus confirming that Reinke's edema is a pathological condition mainly characterized by a chronic fibrotic process, coupled with an excessive extracellular matrix deposition and a strong collagen fibres disarrangement, a high fibroblasts proliferation rate $^{1,8,9,19}$ and fibronectin release, compared to the normal vocal fold architecture, as shown in the negative control (Figure $3 \mathrm{~b}$ ).

\section{Discussion}

Reinke's edema is a pathological condition characterized by a chaotic distribution of short connective fibres and excessive presence of ECM in the Reinke's space. This means that an optimal relation between the arrangement of elastic/collagenous fibres and the amount of ECM is essential for a normal vibratory and phonatory function. ${ }^{22}$ Reinke's edema shows a total structural disarrangement of the collagen fibres architecture in the sub-epithelial layer of the lamina propria, with consequent loss of integrity of the vocal fold function during phonation, main cause of dysphonia. ${ }^{1,8,9,19,20}$ The lamina propria is formed by a wide variety of extracellular components, such as elastic and collagen fibres, glycosaminoglycans, glycoproteins and water. These fibres (mainly collagen type I and III) are concentrated in the superficial layer, closely to the basal membrane of the epithelium (organized in a highly defined network of intertwined fibres, forming a wicker basket) and in the deep layer, more densely intertwined, located parallel to the thyroarytenoid muscle. ${ }^{3,6}$ The lamina propria is also formed by three different cell populations: in the deep layer, fibroblasts (due to the constitutive epithelial regeneration and repair); in the superficial layer, myofibroblasts and macrophages (involved in the inflammatory response to inhalants). ${ }^{6}$ Fibroblasts produce elastic and collagenous fibres as well as intercellular components, including glycosaminoglycan, mucopolysaccarides, elastin and fibronectin. ${ }^{21,22}$ Fibroblasts contribute in maintaining the correct viscosity and elasticity of the vocal folds, so that any structural and functional alteration of the vocal folds is directly related to the fibroblast shape and biological activity.

Reinke's edema is also characterized by an altered vascular permeability resulting in an increase of exudative fluid in the extracellular compartment (main cause of vocal fold swelling), absence of neo-angiogenetic process, high fibroblast proliferation rate, chaotic disarrangement of collagenous and elastic fibres, excessive deposition of extracellular matrix and a chronic inflammatory infiltration, with a strong macrophages activation, especially in the basal membrane of the vocal

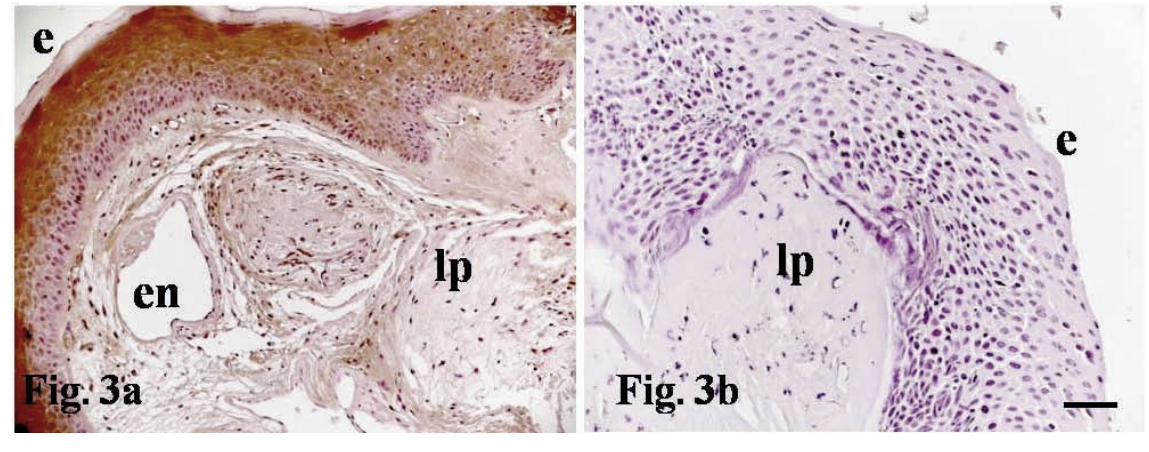

Figure 3. a. Micrograph of representative fibronectin immunostaining in sections of Reinke's edema. Fibronectin reveals a moderate expression level in the mucosal epithelium (e), while a very strong positivity in the lamina propria (lp) and a relevant immunoreactivity on the vessel endothelium (en). b. Micrograph of human normal vocal fold, considered as negative control. Bar: $25 \mu \mathrm{m}$.

fold. ${ }^{1,8,9,19}$

HGF is a growth factor that has a major role in adult organ regeneration and in wound healing, with a strong anti-fibrotic action, leading to a functional fibroblasts alteration and a reduction in collagen and fibronectin release $\mathrm{e}^{16}$ by fibroblasts in Reinke's space. The latter activity leads to morphological and functional changes in this cell population. ${ }^{17,18}$ Hirano et al. ${ }^{17}$ described the presence of HGF in the Reinke's space, responsible for the increase of hyaluronic acid and decrease of collagen I by fibroblasts in wound repairing processes, without affecting the fibronectin deposition. HGF also induces morphological changes in the fibroblasts from an oval to a stellate shape, including a major development in the Golgi apparatus and rough endoplasmic reticulum. ${ }^{17}$ In our observations, we found a moderate HGF expression in the superficial and intermediate layers of the human vocal fold lamina propria, as revealed in the endothelial cells of the human vocal fold, whereas a weak level was observed in the basal membrane. This confirms that anti-fibrotic activity of this factor, in contrast with the collagen production, is stimulated by the fibroblasts present in the inflammatory area of Reinke's edema, without affecting the fibronectin deposition in the lamina propria. MIB-1 (anti-Ki67) in our experiments showed, on the contrary, a weak expression level only in the basal membrane, but it was totally absent in the intermediate and deep layers of the lamina propria, as well as in the vessel endothelium, thus suggesting that only the basal membrane is actively involved in the repairing process with a high regeneration capacity, coupled with a high fibronectin deposition, necessary in the reconstitution of the intercellular connections, following the inflammatory infiltration. Fibronectin, on the other hand, remained strongly expressed in the superficial and intermediate layers of the lamina propria, with a remarkable level detected in the vessel endothelium. It also showed a moderate immunostaining in the epithelial layer of the human vocal fold, thus confirming its functional role in the repairing process during the inflammatory response.

However, Reinke's edema rarely tends to form precancerous lesions: the absence of a neo-angiogenic network (due to a generally altered vessel permeability and endothelial junctions), the chronic inflammatory infiltration, the presence of edematous tissue with a total disarrangement of collagenous and elastic fibres, and the high fibroblastic proliferation (in contrast to the anti-fibrotic activity of HGF) do not allow the cancer cells spreading out, which require a more favorable microenvironment to their growth and transformation.

Finally, our study revealed that MIB-1 (antiKi67) should not be considered a reliable prognostic factor, although more investigations are necessary to clarify the biological and functional role of MIB-1 and HGF in the development and maintenance of Reinke's edema.

\section{References}

1. Thibeault SL. Advances in our understanding of the Reinke space. Curr Opin Otolaryngol Head Neck Surg 2005;13:148151.

2. Volic SV, Klapan I, Seiwerth S, Ibrahimpasic T. Extracellular Matrix of Reinke's Space in some pathological conditions. Acta Otolaryngol 2004;124:505-8.

3. Claassen H, Werner JA. Fibre differentiation of the human laryngeal muscles using the inhibition reactivation myofibrillar ATPase technique. Anat Embryol (Berl) 1992;186:341-6.

4. Madruga de Melo EC, Lemos M, Aragão Ximenes Filho J, Sennes LU, Nascimento 
Saldiva PH, Tsuji DH. Distribution of collagen in the lamina propria of the human vocal fold. Laryngoscope 2003;113:2187-91.

5. Sato K. Reticular fibres in the vocal fold mucosa. Ann Otol Rhinol Laryngol 1998; 107:1023-8.

6. Kutta H, Knipping S, Claassen H, Paulsen F. Functional anatomy of the larynx from clinical viewpoints. Part I: development, laryngeal skeleton, joints, insertion structures, musculature. HNO 2007;55:583-98.

7. Dikkers FG, Nikkels PG. Lamina propria of the mucosa of benign lesions of the vocal folds. Laryngoscope 1999;109:1684-9.

8. Sato K, Hirano M, Nakashima T. Electron microscopic and immunohistochemical investigation of Reinke's edema. Ann Otol Rhinol Laryngol 1999;108:1068-72.

9. Pastuszek P, Krecicki T, Zalesska-Krecicka M, Jelen M, Rak J, Krajewska B. Histological and electron microscopic investigation of Reinke's edema. Pol J Pathol 2003;54:61-4.

10. Remacle M, Degols JC, Delos M. Exudative lesions of Reinke's space. An anatomopathological correlation. Acta Otorhino- laryngol (Belg) 1996;50:253-64.

11. Dikkers FG, Nikkels PG. Benign lesions of the vocal folds: histopathology and phonotrauma. Ann Otol Rhinol Laryngol 1995; 104:698-703.

12. Marcotullio D, Magliulo G, Pietrunti S, Suriano M. Exudative laryngea diseases of Reinke's space: a clinicohistopathological framing. J Otolaryngol 2002;31:376-80.

13. Dikkers FG, Hulstaert CE, Oosterbaan JA, Cervera-Paz FJ. Ultrastructural changes of the basement membrane zone in benign lesions of the vocal folds. Acta Otolaryngol 1993;113:98-101.

14. Gray SD, Hammond E, Hanson DF. Benign pathologic responses of the larynx. Ann Otol Rhinol and Laryngol 1995;104:13-8.

15. Artico M, Bronzetti E, Lo Vasco VR, Ionta B, Alicino V, D’Ambrosio A, Magliulo G. Immunohistochemical profile of various neurotransmitters, neurotrophins and MIB-1 in cholesteatomas of the petrous bone. Mol Med Rep 2008;1:347-53.

16. Comoglio PM. Structure, biosynthesis and biochemical properties of the HGF receptor in normal and malignant cells. EXS
1993;65:131-65.

17. Hirano S, Bless DM, Del Rio AM, Connor NP, Ford CN. Therapeutic potential of growth factors for aging voice. Laryngoscope 2004;114:2161-7.

18. Hirano S, Bless DM, Massey RJ, Hartig GK, Ford CN. Morphological and functional changes of human vocal fold fibroblasts with hepatocyte growth factor. Ann Otol Rhinol Laryngol 2003;112:1026-33.

19. Volic SV, Ibrahimpasic T. Normal and pathologic structure of vocal fold Reinke's space. Acta Clin Croat 2004;43:7-11.

20. Sakae FA, Imamura MD, Sennes L, Mauad T, Saldiva P, Tsuji DH. Disarrangement of collagen fibers in Reinke's edema. Laryngoscope 2008;118:1500-3.

21. Awd Allah RS, Dkhil MA, Farhoud E. Fibroblast in the human vocal fold mucosa: an ultrastructural study of different age groups. Singapore Med J 2009;50:201-7.

22. Hirano M, Sato K, Nakashima T. Fibroblasts in human vocal fold mucosa. Acta Otolaryngol 1999;119:271-6. 